\title{
Utility of Pacemaker with Sleep Apnea Monitor to Predict Left Ventricular Overload and Acute Decompensated Heart Failure
}

João Bicho Augusto MDa,b,c Susana Antunes ${ }^{a}$, João Baltazar Ferreira MDa, Daniel Faria $\mathrm{MD}^{\mathrm{a}}$, David Roque MDa, Marco Beringuilho MDa, Hilaryano Ferreira MDa, Inês Fialho $\mathrm{MD}^{\mathrm{a}}$, Mariana Faustino $\mathrm{MD}^{\mathrm{a}}$, Nuno Cabanelas $\mathrm{MD}^{\mathrm{a}}$, Ana Rita Ferreira $\mathrm{MD}^{\mathrm{a}}$, Hugo Vasconcelos MDa, Miguel Borges Santos MDa, António Freitas MDa, Francisco Madeira MDa, Victor Gil MD, PhD ${ }^{\mathrm{a}, \mathrm{d}}$, Carlos Morais MD

a Cardiology Department, Hospital Professor Doutor Fernando Fonseca, Amadora, Portugal

b Institute of Cardiovascular Science, University College London, London, United Kingdom

${ }^{c}$ Barts Heart Centre, St Bartholomew's Hospital, London, United Kingdom

d Cardiovascular Department, Hospital dos Lusíadas, Lisbon, Portugal

Running title: Pacemaker with sleep apnea monitor

Text word count: 2,342

Corresponding author João Bicho Augusto, MD

Institute of Cardiovascular Science, University College London, Roger Williams Building, 69-75 Chenies Mews, London, WC1E 6HX

T: 0044-207-6796464; F: 0044-207-6796463; E: joao.augusto.18@ucl.ac.uk

Funding This study is funded by an investigator-led research grant from LivaNova. LivaNova had no role in the study beyond funding.

Conflicts of Interest J.B.A. reports grant supports by LivaNova. The other authors have no conflicts of interest to declare. 


\section{Abstract}

Pacemakers with sleep apnea monitor (SAM) provide an easy tool to assess obstructive sleep apnea over long periods of time. The link between respiratory disturbances at night and the incidence of acute decompensated heart failure (ADHF) is not well established. We aimed at (i) determining the ability of SAM pacemakers to evaluate the extent of left ventricular overload and (ii) assess the impact of respiratory disturbances at night on the occurrence of ADHF over 1-year of follow-up. We conducted a single-center prospective study. Consecutive patients with SAM pacemakers were comprehensively assessed. SAM automatically computes a respiratory disturbance index (RDI, apneas/hyponoeas per hour - $\mathrm{AH} / \mathrm{h}$ ) in the previous night and the percentage of nights with $\mathrm{RDI}>20 \mathrm{AH} / \mathrm{h}$ in the previous 6 months. 37 patients were included ( $79.3 \pm 11.2$ years, $46 \%$ males). A high RDI in the previous night and a higher \%nights with increased RDI were associated with increased NT-proBNP values ( $p=0.008$ and $p=0.013$, respectively) and were the sole predictors of increased non-invasive pulmonary capillary wedge pressures (PCWP) in the morning of assessment $(p=0.031$ and $p=0.044$, respectively). ROC curve analysis revealed an area under the curve of $0.804(\mathrm{Cl} 95 \% 0.656-0.953, \mathrm{p}=0.002)$ for \%nights with $\mathrm{RDI}>20 \mathrm{AH} / \mathrm{h}$ in the prediction of high PCWP. Patients with $>12.5 \%$ of nights with $\mathrm{RDI}>20 \mathrm{AH} / \mathrm{h}$ tended to have more ADHF during follow-up (log-rank $\mathrm{p}=0.067$ ). In conclusion, a high burden of apneas/hypopneas at night is associated with elevated NT-proBNP and PCWP values and an increased risk of ADHF over 1 year. These patients might benefit from early tailored clinical management.

Keywords: Sleep apnea; pacemaker; pulmonary wedge pressure; heart failure 


\section{Introduction}

Sleep-related breathing disorders are known to have a high prevalence in patients suffering from cardiovascular (CV) diseases (1)(2)(3). Obstructive sleep apnea (OSA) is highly prevalent in heart failure (HF, 50-70\%) and has a significant impact on prognosis if left untreated (4). Currently, some pacemakers (PMs) incorporate a sleep apnea monitor (SAM) which allows monitorization over long periods (months) and can estimate the respiratory disturbance index during sleep (5). However, there is a lack of evidence correlating the severity of apnea/hypopnea events at night and its repercussion on left ventricular (LV) overload and subsequent risk of acute decompensated HF (ADHF). Taking advantage of the high incidence of OSA in HF (6) and the fact that OSA may affect up to half of the pacemaker population (7), this study aims at (i) determining the ability of the SAM function in patients with conventional PM to evaluate the extent of left ventricular (LV) overload using NTproBNP (brain natriuretic peptide) and non-invasive measurements of pulmonary capillary wedge pressure (PCWP) and (ii) assess the impact of respiratory disturbances at night on the occurrence of ADHF over 1-year of follow-up.

\section{Methods}

This is a prospective single-center study (Hospital Professor Doutor Fernando Fonseca) in consecutive patients evaluated at baseline in a PM clinic who had a singleor a dual-chamber anti-bradycardia PM with an incorporated SAM algorithm. The study has been approved by the local ethics committee and regulatory health authority. Informed consent was obtained for all patients. The study was conducted in accordance with the Declaration of Helsinki, Good Clinical Practice, and all applicable 
laws and regulations. All adverse events occurring during the course of the study were reported, followed up, and reviewed within the common clinical practice.

Patients with single or dual chamber PMs with incorporated SAM (REPLY 200TM, KORA 250TM and KORA 100TM SR/DR, LivaNova®, France) were consecutively included between October 2016 and January 2017, regardless of symptoms suggesting SA. Patients' baseline assessment was performed in the morning for all cases (between 8:30 am and 1:00 pm). We excluded patients who (i) were aged $<18$ years, (ii) had a known significant (moderate or severe) valve disease, (iii) congenital cardiac disease and/or (iv) moderate or severe pulmonary hypertension. At the inclusion consultation demographic and clinical data were recorded, including comorbidities. Fasting blood samples were collected and processed locally on the same day. A transthoracic echocardiogram was also performed on the same morning: structural and functional LV and RV data were collected, including pulsed wave Doppler from the LV inflow tract and transmitral E/e' ratio (e' as a mean of both septum and lateral annulus in Tissue Doppler imaging analysis). Left atrial volume and mean right atrial pressure were also recorded. PCWP is an indirect estimate of left atrial pressure (8) and represents an alternative measure to LV end-diastolic pressure, which reflects LV filling pressures. Applying the Nagueh formula, PCWP was extrapolated non-invasively from the transmitral E/e' ratio (9):

$$
\mathrm{PCWP}=1.24 \times \frac{E}{e^{\prime}}+1.9 \mathrm{mmHg}
$$

PM records were assessed for percentage of atrial and ventricular pacing and nocturnal atrioventricular blocks (second- and third-degree blocks according to the number of blocked atrial events as determined by the PM algorithm). The SAM algorithm has been previously described (10). A PM sensor measures each respiratory cycle's amplitude and period, and the minute-ventilation value is calculated (Figure 
1A). The SAM checks a specific 5-hour period during night, which was prespecified in our study as between 0.00 and $5.00 \mathrm{am}$. The SAM algorithm detects: apneas (absence of a significant respiratory cycle for $>10$ seconds) and hypopneas (sustained, $>10$ seconds, reduction of the respiratory amplitude by at least $50 \%$ compared to the mean minute ventilation of preceding validated respiratory cycles). A respiratory disturbance index $(\mathrm{RDI})$ is then automatically computed as the mean number of respiratory events detected per hour of sleep. We recorded the RDI of the night prior to the assessment in clinic as well as the percentage of nights with $\mathrm{RDI}>20$ apneas/hypopneas per hour $(\mathrm{AH} / \mathrm{h})$ in the 6 months before the assessment; this cut-off has been shown to be a marker of severe OSA (5). A representative patient is shown in Figures 1B and 1C.

All patients were followed for 1 year from the day of initial assessment. Followup was performed by one clinician (J.B.F.) who was blinded to the RDI data. Followup data was obtained from patients' hospital electronic medical records (including outpatient visits and hospital admissions) and by enquiring the national health data system or, if any was unavailable, by telephone interview. Primary outcome was defined as admission for ADHF. Secondary outcomes included non-cardiovascular death, cardiovascular death, and a composite of all-cause mortality and ADHF.

Statistical analysis was performed on SPSS version 22.0 (SPSS Inc., Chicago, IL, USA). Discrete variables are presented as absolute frequencies with percentages; continuous variables are presented as mean \pm standard deviation (SD) if normally distributed, otherwise as median and interquartile ranges (IQR). Comparisons between groups were performed using Students' t-test or Mann-Whitney U test for parametric and nonparametric data, respectively; categorical variables were compared using Fisher's exact test. Logistic regression was conducted (using the 'enter' method) to assess which factors predicted increased PCWP $(>16 \mathrm{mmHg})$. The ability of the above 
significant factors to predict increased PCWP was then assessed using Receiver Operating Characteristic (ROC) curve analysis. An optimal cut-off to predict high PCWP was determined by the best trade-off between sensitivity and specificity as assessed by Youden's index. Kaplan-Meier survival analysis was used to plot time-toevent curves for 1 -year after discharge according to the proportion of nights with $\mathrm{RDI}>20 \mathrm{AH} / \mathrm{h}$ prior to the baseline assessment (as this provides a representative overview of the breathing pattern during sleep). Comparisons were performed using the log-rank test. Two-sided $p$-values $<0.05$ were considered significant.

\section{Results}

A total of 37 patients were eligible for final analysis. Mean age was $79.3 \pm 11.2$ years, with $86.5 \%$ being at least 65 years-old and $45.9 \%$ were males. Baseline characteristics are summarized in Table 1. Most patients had a dual-chamber PM $(70.3 \%, \mathrm{n}=26)$. The sampled population was overweight with a mean BMI of $27.1 \mathrm{~kg} / \mathrm{m}^{2}$ (18 subjects were overweight and 8 were obese). Day sleepiness (Epworth sleepiness score $>10)$ was found in $22 \%(n=8)$ patients. Three patients had known OSA, 2 of them were already receiving treatment with continuous positive airway pressure (CPAP). Epworth scores were 3 times higher when was $\mathrm{RDI}>20 \mathrm{AH} / \mathrm{h}$ in the previous night (14.0 [4.0-15,0] vs $5.0[1.0-7.0]$ in patients with $\mathrm{RDI} \leq 20 \mathrm{AH} / \mathrm{h}, \mathrm{p}=0.073)$.

Higher RDI values in the previous night values were associated with elevated PCWP and NT-proBNP values in the morning (both $r=0.43, p=0.008$ ). Similarly, higher proportions of nights with $\mathrm{RDI}>20 \mathrm{AH} / \mathrm{h}$ were associated with elevated PCWP $(r=0.43$, $\mathrm{p}=0.007)$ and NT-proBNP values in the morning $(r=0.41, p=0.013)$. Patients with $\mathrm{RDI}>20 \mathrm{AH} / \mathrm{h}$ in the previous night had significantly higher values of PCWP (26.3 [21.7 - 31.3] vs $15.5[12.6-20.1] \mathrm{mmHg}, \mathrm{p}=0.006)$ but NT-proBNP values were not 
significantly different (1856 [412 - 2750] vs 820 [186 - 1485]pg/mL, $p=0.193)$. Patients with over $27 \%$ of nights ( $75^{\text {th }}$ percentile) with $\mathrm{RDI}>20 \mathrm{AH} / \mathrm{h}$ over 6 months had higher values of PCWP $(26.3[19.3-30.7]$ vs 15.5 [12.6 - 22.3]mmHg, $\mathrm{p}=0.023)$ and NTproBNP (2750 [736 - 5119] vs $540[186-1413] p g / m L, ~ p=0.040)$. Univariate logistic regression analysis identified RDI in the previous night and proportion of nights with $\mathrm{RDI}>20 \mathrm{AH} / \mathrm{h}$ as predictors of increased PCWP ( $p=0.031$ and $p=0.044$, respectively). As RDI (in the previous night / six months) was the sole predictor of high PCWP, multivariable analysis was not performed.

ROC curve analysis showed a high area under the curve for both RDI in the previous night (AUC $0.812,95 \% \mathrm{Cl} 0.667-0.957, \mathrm{p}=0.001$ ) and proportion of nights with $\mathrm{RDI}>20 \mathrm{AH} / \mathrm{h}(\mathrm{AUC} 0.804,95 \% \mathrm{Cl} 0.656-0.953, \mathrm{p}=0.002)$ in the prediction of high PCWP (Figure $2 \mathrm{~A}$ ). An RDI $>8 \mathrm{AH} / \mathrm{h}$ and $>12.5 \%$ of nights with $\mathrm{RDI}>20 \mathrm{AH} / \mathrm{h}$ were the optimal cut-offs for predicting high PCWP (Figure 2A). Follow-up was available for all patients. At 1-year follow-up there were 3 admissions for ADHF (8.1\%), 1 admission following stroke (2.7\%), 3 cardiovascular deaths (8.1\%) and 1 non-cardiovascular death $(2.7 \%)$. Proportion of nights with $\mathrm{RDI}>20 \mathrm{AH} / \mathrm{h}$ were used in survival analysis. Kaplan-Meier survival analysis identified a tendency to higher incidence of ADHF in patients who had over $12.5 \%$ of nights with $\mathrm{RDI}>20 \mathrm{AH} / \mathrm{h}$ at baseline (log-rank $\mathrm{p}=0.067$, Figure 2B); the incidences of cardiovascular death, non-cardiovascular death and composite secondary outcome (all-cause mortality and ADHF) were not significantly higher for this group of patients.

\section{Discussion}

This study has explored the role of PMs with SAM algorithm in the prediction of high LV filling pressures and the occurrence of ADHF at 1-year follow-up. Our main 
findings are: (i) a high RDI in the previous night and a higher proportion of nights with increased RDI can predict increased LV filling pressures (as assessed by non-invasive PCWP and NT-proBNP) and (ii) a high burden of apneas/hypopneas at night is associated with an increased risk of ADHF.

The prevalence of OSA in patients with PM has been shown to be much higher than in the general population (59 vs $21 \%$ ) (7). This higher prevalence can be explained by the association of OSA with prominent and potentially symptomatic bradycardia events, which in turn might lead to PM implantation while OSA is unknown (11). Given that more than half of patients with PM are at risk of having OSA, close monitoring and management of these patients is warranted. RDI is an easy to obtain marker in patients who already have a PM with the SAM algorithm and is a marker of severe OSA (5). Indeed, the SAM algorithm allows monitorization for long periods thus providing more representative assessment of OSA than that of a single night.

We found that a higher RDI at night was associated with high LV filling pressures (high NT-proBNP and PCWP), which can shed a light on the heart failure pathophysiology in this group of patients. OSA has been described as a cause of LV diastolic dysfunction (12) and this can be a result of a (mal)adaptive response to cardiac overload. Repetitive changes in heart rate, cardiac output as well as in systemic and pulmonary arterial pressures have been described with OSA (13) and these can occur as a consequence of increased sympathetic nervous system activity. The active LV relaxation can also be impaired: diseases with increased pressure overload (such as systemic hypertension) have reduced levels of sarcoplasmic reticulum calcium ATPase pump (14). In addition, multiple cellular signals can cause LV hypertrophy and interstitial fibrosis, and increase passive LV stiffness as well (15). 
A high burden of apneas/hypopneas at night was associated with an increased risk of admission for ADHF at 1-year follow-up. Interestingly, baseline NT-proBNP and non-invasive PCWP values were high and associated with OSA. This highlights the relevance of OSA on the chronic hemodynamic stress in these patients. One might ask whether OSA is the cause of $\mathrm{HF}$, or if there is an underlying undiagnosed $\mathrm{HF}$ that might be the cause of OSA (16). The latter could be explained by a fluid displacement in the recumbent position during sleep, which might lead to increased pressure in the peripharyngeal tissues and, consequently, cause OSA. Nonetheless, regardless of what comes first (OSA or HF), it is fair to conclude that OSA is clearly a contributor to ADHF during follow-up and hence should be properly monitored and addressed.

The link between OSA and HF is not well established and the LV diastolic dysfunction does not seem to be the sole mechanism of acute HF. Other mechanisms are likely involved, including inflammation, hypoxia, oxidative stress, endothelial dysfunction and other neurohormonal pathways (16)(17). Importantly, treatment with CPAP has been shown to improve LVEF in patients with HF and severe OSA (18). Hence, a timely detection of OSA and prompt treatment initiation might prevent LVEF deterioration. As more than half of patients with PM have OSA, cardiologists should be more aware of this entity. In the presence of a high burden of nights with OSA, further clinical assessment and appropriate treatment should be provided.

Other makers of PMs also provide apnea monitoring, but such algorithms have not been studied for LV filling pressure assessment. Some cardiac resynchronization therapy (CRT) devices track fluid accumulation in the lungs/thoracic cavity by means of intrathoracic impedance (19) - with fluid accumulation there is a decrease in intrathoracic impedance. A strong relationship has been described between intrathoracic impedance and transthoracic conductance, the latter corresponding to the 
inverse of transthoracic impedance (used in SAM) (20). However, CRTs are only limited to a selected subgroup of patients who already has HF. In contrast, PMs with SAM could open a new window of opportunity by monitoring a larger population at risk of ADHF. Having access to the absolute transthoracic impedance values in these PMs could however provide more meaningful data for patient management.

Limitations include the single center nature of the study, the absence of a control population and the relatively small sample size, although the latter represents a muchselected group of patients without significant comorbidities (that could act as confounders). The cause of the sleep apnea (obstructive and/or central) was not established. Only one type of SAM was studied. We believe that algorithms based on similar physiological assumptions should yield similar results, but further studies are needed. PCWP was estimated and not directly measured, possibly contributing to a misrepresentation of LV filling pressures. Nevertheless, this physiological inference is derived from the E/e' ratio (also a marker of LV pressures) and is corroborated by the concomitant increase in NT-proBNP and higher rates of ADHF. A formal causal association between RDI and heart failure cannot be fully ascertained in this study, but our findings suggest that a high burden of apneas/hypopneas contributes to ADHF.

In conclusion, patients with PM have a high prevalence of OSA which is usually overlooked in clinical practice. We have shown that the respiratory disturbance events measured at night with SAM are associated with high LV filling pressures (as assessed by NT-proBNP and non-invasive PCWP) and an increased incidence of ADHF over a year. When the burden of apneas/hypopneas at night is high, patients might benefit from further clinical assessment, although more studies are needed to evaluate the impact of such a strategy on clinical outcomes. 


\section{References}

1. Somers VK, White DP, Amin R, Abraham WT, Costa F, Culebras A, Daniels S, Floras JS, Hunt CE, Olson LJ, Pickering TG, Russell R, Woo M, Young T. Sleep Apnea and Cardiovascular Disease: an American Heart Association/American College of Cardiology Foundation Scientific Statement from the American Heart Association Council for High Blood Pressure Research Professional Education Committee, Council on Clinical Cardiology, Stroke Council, and Council on Cardiovascular Nursing. J Am Coll Cardiol 2008;52:686-717.

2. Koehler U, Schäfer H. Is obstructive sleep apnea (OSA) a risk factor for myocardial infarction and cardiac arrhythmias in patients with coronary heart disease (CHD)? Sleep 1996;19:283-286.

3. Gami AS, Pressman G, Caples SM, Kanagala R, Gard JJ, Davison DE, Malouf JF, Ammash NM, Friedman PA, Somers VK. Association of atrial fibrillation and obstructive sleep apnea. Circulation 2004;110:364-367.

4. Oldenburg O, Lamp B, Faber L, Teschler H, Horstkotte D, Töpfer V. Sleepdisordered breathing in patients with symptomatic heart failure: a contemporary study of prevalence in and characteristics of 700 patients. Eur J Heart Fail 2007;9:251-257.

5. Defaye P, de la Cruz I, Martí-Almor J, Villuendas R, Bru P, Sénéchal J, Tamisier R, Pépin JL. A pacemaker transthoracic impedance sensor with an advanced algorithm to identify severe sleep apnea: The DREAM European study. Heart Rhythm 2014;11:842-848.

6. Shahar E, Whitney CW, Redline S, Lee ET, Newman AB, Nieto FJ, O'Connor GT, Boland LL, Schwartz JE, Samet JM. Sleep-disordered Breathing and Cardiovascular Disease Cross-sectional Results of the Sleep Heart Health Study. Am 
J Respir Crit Care Med 2001;163:19-25.

7. Garrigue S, Pépin JL, Defaye P, Murgatroyd F, Poezevara Y, Clémenty J, Lévy P. High prevalence of sleep apnea syndrome in patients with long-term pacing: the European Multicenter Polysomnographic Study. Circulation 2007;115:1703-1709.

8. Nagy Al, Venkateshvaran A, Dash PK, Barooah B, Merkely B, Winter R, Manouras A. The pulmonary capillary wedge pressure accurately reflects both normal and elevated left atrial pressure. Am Heart J 2014;167:876-883.

9. Nagueh SF, Middleton KJ, Kopelen HA, Zoghbi WA, Quiñones MA. Doppler Tissue Imaging : A Noninvasive Technique for Evaluation of Left Ventricular Relaxation and Estimation of Filling Pressures. J Am Coll Cardiol 1997;30:1527-1533.

10. Defaye P, Pépin JL, Poezevara Y, Mabo P, Murgatroyd F, Lévy P, Garrigue S. Automatic Recognition of Abnormal Respiratory Events During Sleep by a Pacemaker Transthoracic Impedance Sensor. J Cardiovasc Electrophysiol 2004;15:1034-1040.

11. Gula LJ, Krahn AD, Skanes AC, Yee R, Klein GJ. Clinical relevance of arrhythmias during sleep: guidance for clinicians. Heart 2004;90:347-352.

12. Arias MA, García-Río F, Alonso-Fernández A, Mediano O, Martínez I, Villamor J. Obstructive sleep apnea syndrome affects left ventricular diastolic function: effects of nasal continuous positive airway pressure in men. Circulation 2005;112:375-383.

13. Podszus T, Mayer J, Penzel T, Peter JH, von Wichert P. Nocturnal hemodynamics in patients with sleep apnea. Eur J Respir Dis Suppl 1986;146:435442.

14. Arai M, Alpert NR, MacLennan DH, Barton P, Periasamy M.. Alterations in sarcoplasmic reticulum gene expression in human heart failure. A possible mechanism 
for alterations in systolic and diastolic properties of the failing myocardium. Circ Res 1993;72:463-469.

15. Lorell BH, Carabello BA. Left ventricular hypertrophy: pathogenesis, detection, and prognosis. Circulation 2000;102:470-479.

16. Kasai T, Bradley TD. Obstructive Sleep Apnea and Heart Failure Pathophysiologic and Therapeutic Implications. J Am Coll Cardiol 2011;57:119-127.

17. Savransky V, Nanayakkara A, Li J, Bevans S, Smith PL, Rodriguez A, Polotsky VY. Chronic Intermittent Hypoxia Induces Atherosclerosis. Am J Respir Crit Care Med 2007;175:1290-1297.

18. Kaneko Y, Floras JS, Usui K, Plante J, Tkacova R, Kubo T, Ando S, Bradley TD. Cardiovascular Effects of Continuous Positive Airway Pressure in Patients with Heart Failure and Obstructive Sleep Apnea. N Engl J Med 2003;348:1233-1241.

19. Whellan DJ, Ousdigian KT, Al-Khatib SM, Pu W, Sarkar S, Porter CB, Pavri BB, O'Connor CM, PARTNERS Study Investigators. Combined heart failure device diagnostics identify patients at higher risk of subsequent heart failure hospitalizations: results from PARTNERS HF (Program to Access and Review Trending Information and Evaluate Correlation to Symptoms in Patients With Heart Failure) study. J Am Coll Cardiol 2010;55:1803-1810.

20. Malfatto G, Villani A, Rosa FD, Rella V, Oldani M, Giglio A, Facchini M, Parati G. Correlation between trans and intra-thoracic impedance and conductance in patients with chronic heart failure. J Cardiovasc Med (Hagerstown) 2016;17:276-282. 


\section{Figure Legends}

Figure 1. Illustration of the pacemaker with sleep apnea monitor is shown (A). The voltage $(\mathrm{U})$ between the ring electrode and the pacemaker as well as the current (I) injected between the electrode tip and pacemaker are measured. Transthoracic impedance $(Z)$ is calculated as the ratio between $U$ and I. For each respiratory cycle, the sensor will determine both amplitude and period of minute-ventilation (MV). Apnea is defined as absence of a significant respiratory cycle for $>10$ seconds. Hypopnea is defined as a reduction of at least $50 \%$ in the average amplitude of the previous $8 \mathrm{MVs}$ over 10 seconds. Sleep apnea monitoring data is shown for patient \#33 (B and C). The number of disturbed respiratory events per hour is shown (A, purple bars) during the last night (between 00:00 - 05:00) in parallel with the heart rate curve. In this case, the total number of disturbed events overnight was 225 , giving a respiratory disturbance index (RDI) of 45 (225 events/5 hours). RDI is also recorded for each night since the last follow-up ( $B$, up to the last 6 months). The percentage of nights since the last follow-up with RDI $>20$ events/hour is also noted.

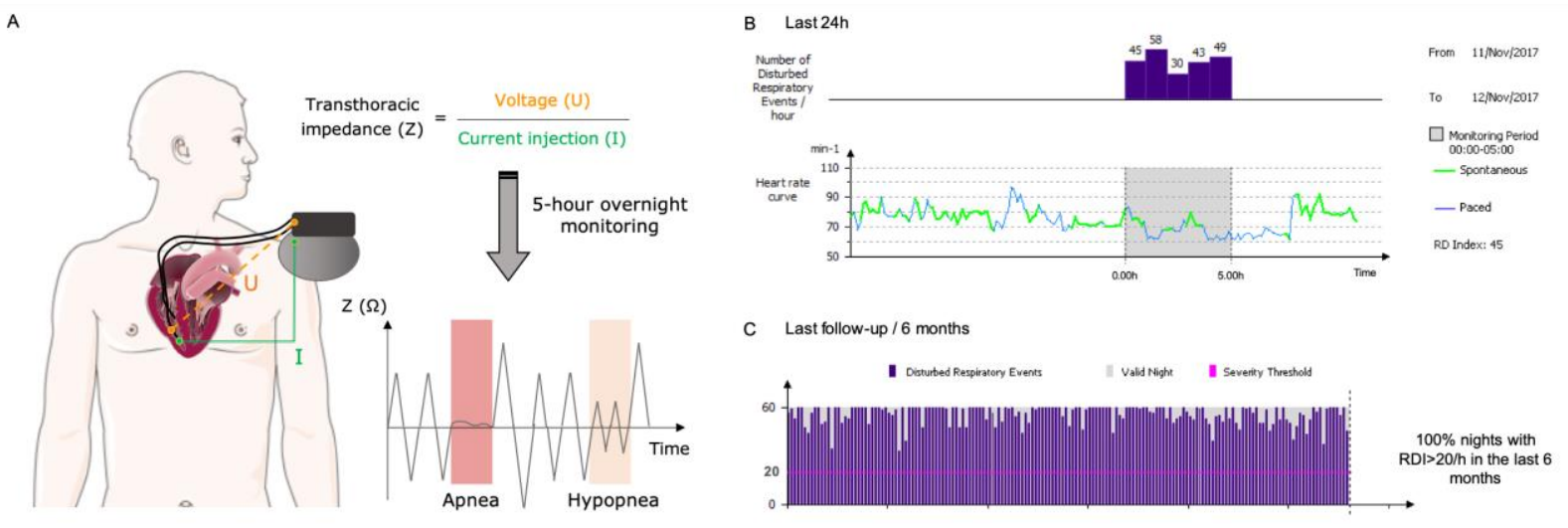


Figure 2. ROC curves of $\mathrm{RDI}$ in the previous night and proportion of nights with $\mathrm{RDI}>20 \mathrm{AH} / \mathrm{h}$ in the last 6 months for prediction of PCWP $>16 \mathrm{mmHg}(\mathrm{A})$. Kaplan-Meier curves of survival free of acute decompensated heart failure (HF) over 1-year of followup according to the proportion of nights with $\mathrm{RDI}>20 \mathrm{AH} / \mathrm{h}$ at baseline (B).

A

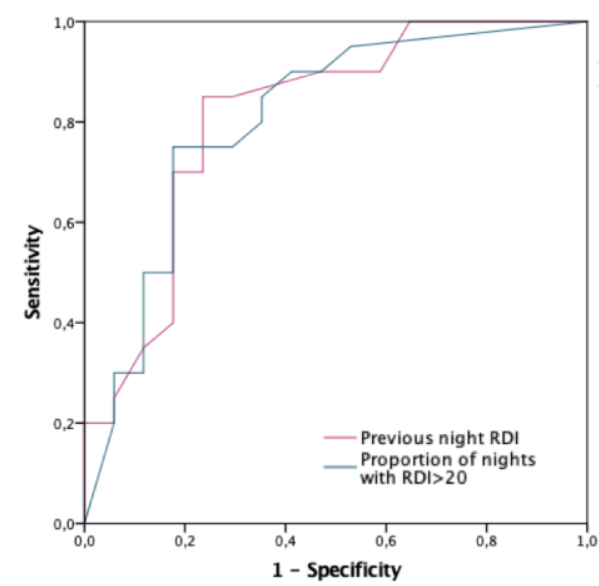

B

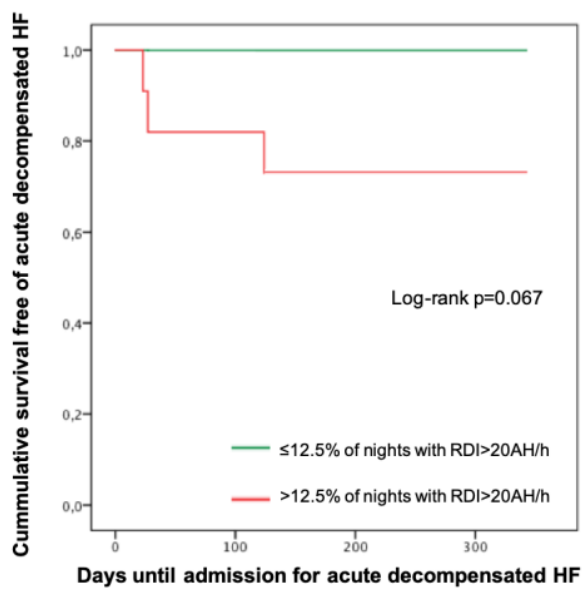

\begin{tabular}{|lccccc|}
\hline & $\begin{array}{c}\text { Optimal } \\
\text { cut-off }\end{array}$ & Sensitivity & Specificity & NPV & PPV \\
\hline Previous night RDI & $>8 \mathrm{AH} / \mathrm{h}$ & 0.85 & 0.77 & 0.79 & 0.84 \\
& $(0.62-0.97)$ & $(0.50-0.93)$ & $(0.72-0.84)$ & $(0.77-0.89)$ \\
\hline $\begin{array}{l}\text { Proportion of nights } \\
\text { with RDI>20AH/h }\end{array}$ & $>12.5 \%$ & 0.75 & 0.82 & 0.74 & 0.83 \\
& & $(0.51-0.91)$ & $(0.57-0.96)$ & $(0.56-0.86)$ & $(0.63-0.94)$ \\
\hline
\end{tabular}

$\mathrm{AH} / \mathrm{h}$, apneas/hypopneas per hour; AUC, area under the curve; NPV, negative predictive value; PCWP, pulmonary capillary wedge pressure; PPV, positive predictive value; $\mathrm{RDI}$, respiratory disturbance index.

Results presented with $95 \%$ confidence interval. 
Table 1. Baseline characteristics of the study population.

\begin{tabular}{|c|c|}
\hline Age (years) & $79.3 \pm 11.2$ \\
\hline Men & $17(45.9)$ \\
\hline Body mass index $\left(\mathrm{Kg}^{*} \mathrm{~m}^{-2}\right)$ & $27.1 \pm 3.8$ \\
\hline Heart rate (bpm) & $75(70-80)$ \\
\hline Systolic blood pressure $(\mathrm{mmHg})$ & $147(129-170)$ \\
\hline Peripheral capillary oxygen saturation (\%) & $96(95-97)$ \\
\hline Hypertension & $35(94.6)$ \\
\hline Diabetes mellitus & $10(27.0)$ \\
\hline Smoker & $3(8.1)$ \\
\hline Chronic obstructive pulmonary disease & $2(5.4)$ \\
\hline Stroke & $6(16.2)$ \\
\hline Atrial fibrillation & $17(45.9)$ \\
\hline Hypothyroidism & $7(18.9)$ \\
\hline Heart failure & $8(21.6)$ \\
\hline Obstructive sleep apnea & $3(8.1)$ \\
\hline Epworth questionnaire score & $5(1-9)$ \\
\hline Haemoglobin (g/dL) & $13.3(12.7-14.2)$ \\
\hline Creatinine (mg/dL) & $1.0(0.8-1.3)$ \\
\hline Sodium (mmol/L) & $141.0(139.0-142.3)$ \\
\hline Glycated hemoglobin A1C (\%) & $5.6(5.3-6.0)$ \\
\hline Thyroid-stimulating hormone (mU/L) & $1.9(0.9-3.2)$ \\
\hline Total cholesterol (mg/dL) & $168(151-180)$ \\
\hline Low-density lipoprotein (mg/dL) & $75(63-100)$ \\
\hline Uric acid (mg/dL) & $4.7(4.3-6.3)$ \\
\hline C-reactive protein (mg/dL) & $0.3(0.3-0.6)$ \\
\hline Interleukin-6 (pg/mL) & $2.0(2.0-4.5)$ \\
\hline NT-proBNP (pg/mL) & $1040(191-1972)$ \\
\hline Troponin I (ng/mL) & $0.02(0.02-0.02)$ \\
\hline D-dimers ( $\mu \mathrm{g} / \mathrm{L})$ & $623(420-1669)$ \\
\hline \multicolumn{2}{|l|}{ Echocardiogram } \\
\hline Left ventricle end-diastolic volume index $\left(\mathrm{mL} / \mathrm{m}^{2}\right)$ & $42.1(36.4-58.2)$ \\
\hline Left ventricle ejection fraction (\%) & $50(45-55)$ \\
\hline Basal right ventricular diameter (mm) & $34.5(31.7-41.9)$ \\
\hline Right ventricular fractional area change (\%) & $47.0(36.5-57.5)$ \\
\hline Tricuspid annular plane systolic excursion (mm) & $19.6(18.3-23.0)$ \\
\hline Lateral tricuspid S'-wave $(\mathrm{cm} / \mathrm{s})$ & $10.0(8.0-14.0)$ \\
\hline Left atrial end-systolic volume index $\left(\mathrm{mL} / \mathrm{m}^{2}\right)$ & $40.7(28.2-65.6)$ \\
\hline Mean right atrial pressure $(\mathrm{mmHg})$ & $3.0(3.0-8.0)$ \\
\hline
\end{tabular}


Transmitral E/A

$1.2(0.7-2.5)$

Transmitral E/e'

$11.5(9.3-18.8)$

Pulmonary capillary wedge pressure $(\mathrm{mmHg})$

$16.2(13.4-25.2)$

\section{Pacemaker}

Nocturnal atrioventricular blocks

$9(24.3)$

Proportion of time in atrial pacing (\%)

$49(0-86)$

Proportion of time in ventricular pacing (\%)

$77(7-99)$

Previous night respiratory disturbance index (apneas/hypopneas per hour)

$10.0(6.0-19.0)$

Proportion of nights with respiratory disturbance index $>20$ apneas/hypopneas per hour in the last 6 months (\%)

$11.0(1.0-27.0)$

\section{Medication/treatment}

Angiotensin-converting-enzyme inhibitor / angiotensin II receptor blockers

Beta-blockers

Mineralocorticoid receptor antagonists

Loop diuretics

Nocturnal non-invasive ventilation

$2(5.4)$

Continuous variables presented as mean \pm standard deviation or median (interquartile range). 